\title{
Profile of bevacizumab and its potential in the treatment of cervical cancer
}

This article was published in the following Dove Press journal:

OncoTargets and Therapy

19 November 2015

Number of times this article has been viewed

\section{Christine M Fisher \\ Tracey E Schefter}

Department of Radiation Oncology, University of Colorado, Denver, CO, USA
Correspondence: Tracey E Schefter Department of Radiation Oncology, University of Colorado, 1665 Aurora Court, Suite 1032 MS F706, Aurora, CO 80045 USA

Email tracey.schefter@ucdenver.edu

\begin{abstract}
Blocking angiogenesis is an effective antitumor strategy proven in many disease sites. Anti-angiogenic therapies are fulfilling the promise of improved outcomes in cervical cancer as demonstrated in several recent trials. With its overall survival improvement in metastatic or recurrent cervical cancer, a frame shift in the management of these patients has occurred. The US Food and Drug Administration approval of bevacizumab in advanced cervical cancer has led to national guidelines, including the US National Comprehensive Cancer Network guidelines for cervical cancer, including systemic regimens containing bevacizumab as first line combination therapy. Future trials will build on this anti-angiogenesis backbone via targeting additional novel pathways and potentially leading to further improved outcomes in cervical cancer.
\end{abstract}

Keywords: HPV, anti-angiogenic therapy, cervix, arastin, VEGF, metastatic, recurrent

\section{Introduction}

Cervical cancer diagnoses in the US, ${ }^{1}$ and worldwide, ${ }^{2}$ continues to be a major cause of morbidity and mortality among young women. Developing nations are targeting improvements in systemic therapy for cervical cancer to improve the lives of young mothers. ${ }^{3}$ Given the worldwide prevalence of cervical cancer and the associated cost of losing young women, there is a need for better therapies.

Concurrent chemotherapy and radiotherapy are the standard of care for intermediate and locally advanced disease, ${ }^{4}$ yet our ability to successfully manage patients with recurrent, persistent, and metastatic disease remains suboptimal. Given the overlapping toxicities of maximal systemic cytotoxic therapy and radiation therapy as well as continued suboptimal outcomes in this population, an investigation of targeted agents to improve outcomes was undertaken. Herein, we review the potential of anti-angiogenic agents in cervical cancer with current data and updated expert guidelines.

\section{Profile of bevacizumab}

Bevacizumab represents a novel therapeutic strategy targeting the vascular endothelial growth factor (VEGF) pathway. Bevacizumab is a recombinant humanized monoclonal antibody to VEGF. Likely based on the mechanism of pruning vessels, bevacizumab has shown a synergistic effect with multiple chemotherapeutic agents. ${ }^{5}$ Bevacizumab has a role in the treatment of many cancers, including cervical cancer, colorectal cancer, and ovarian cancer, ${ }^{6}$ with approval for multiple indications granted by the US Food and Drug Administration (FDA) last year.

The proposed mechanism of bevacizumab's paradoxical synergy with both chemotherapy and radiation, despite treatment relying on tumor perfusion and free 
radical generation, is explained by the vessel normalization hypothesis whereby anti-VEGF effects normalize the vasculature to improve therapeutic outcomes. ${ }^{7,8}$ Lee et al demonstrated synergy of bevacizumab and radiation in a preclinical study which showed improved oxygenation. ${ }^{9}$ This effect is seen across multiple anti-angiogenic agents. ${ }^{10}$ Current bevacizumab practice includes maintenance therapy in some clinical scenarios, even after progression, ${ }^{11}$ though the lifespan of the vascular pruning is not clear. ${ }^{12}$ Restoration of the vasculature to a more typical phenotype enhances tumor susceptibility to concurrent delivery of chemotherapy and radiation. ${ }^{13}$

Given the intensive previous therapy that persistent and recurrent as well as primarily metastatic cervical cancer patients receive, it is important to be aware of the potential serious side effects. In this population treated with concurrent bevacizumab and chemotherapy, at least $20 \%$ of patients developed fatigue, anorexia, hypertension, hyperglycemia, hypomagnesemia, headache, weight loss, and urinary tract infections. One of the more serious potential side effects, gastrointestinal perforation, was seen in $3.2 \%$ of patients treated with bevacizumab. Prior pelvic radiation was associated with an $8.2 \%$ rate of gastrointestinal-vaginal fistulae with bevacizumab compared to a $0.9 \%$ rate without bevacizumab, suggesting a potential synergistic toxicity. Grade 3 venous thromboembolic events were also noted more frequently in the bevacizumab treated patients. ${ }^{14}$

\section{Biological rationale of blocking angiogenesis in cervical cancer}

Cervical cancer induction is attributable to human papilloma virus (HPV) infection in the vast majority of cases. ${ }^{15} \mathrm{HPV}-$ negative cervical carcinomas represent a distinct and rare subtype in which different biological drivers have yet to be identified. ${ }^{16}$ For the pervasive HPV positive cervical carcinomas, HPV leads to degradation of the tumor suppressor gene, p53. Yang et al looked at the association of HPV-16, E6, and p53 expression in cervical cancer tissues compared to normal epithelium, noting that E6 expression is likely the cause of inactivation of p53 in tumor tissue, supporting the mechanism of tumorigenesis. ${ }^{17}$

Loss of p53 leads to upregulation of hypoxia inducible factor-1 (HIF-1), which is implicated in increasing neovascularization. For cervical cancer in particular, overexpression of HIF-1 has also been identified as an independent negative prognostic marker. ${ }^{18}$ In normal tissue, p53 will downregulate the increasing HIF-1 and VEGF expression, yet this downregulation disappears with the loss of $\mathrm{p} 53 .{ }^{19,20}$
A meta-analysis and clinicopathologic study confirmed on multivariate analysis that HIF-1 alpha overexpression is associated with a hazard ratio of 2.57 , emphasizing the importance of HIF-1 expression as a predictor of outcome in cervical cancer. ${ }^{21}$ Beyond quantifying expression levels, markedly increased risk of cervical cancer has also been associated with the rs2057482 polymorphism in the three untranslated regions of HIF-1 alpha, which may serve as another marker for cervical cancer risk (and potential target for combination therapy). ${ }^{22}$

The long-standing recognition that tumors are highly vascular has led to recent inquiry into the biological drivers of angiogenic stimulation. Anti-VEGF antibody, bevacizumab, was developed to take advantage of this susceptibility. ${ }^{23}$ Tumors recruit the growth of nearby vascular endothelial cells from veins, arteries, and even lymphatic channels, resulting in the formation of new blood vessels (angiogenesis) to support tumor growth. Elevated VEGF expression has been demonstrated in various solid tumors, ${ }^{24,25}$ including cervical cancer. ${ }^{26}$ Increased angiogenesis may also be a marker for enhanced biologic aggressiveness. In one series of 111 women with cervical cancer, high tumor microvascular density was a significant prognostic factor associated with poorer overall survival and local control on multivariate analysis. ${ }^{27}$

VEGF is a dimeric glycoprotein that has been shown to trigger proliferation of endothelial cells, regulate membrane permeability, and promote recruitment of stem cells to sites of neovascularization. ${ }^{28,29}$ This new vessel formation does not happen in a typical robust fashion. Neovascularization from tumors branches out in a haphazard, distorted manner, lacking appropriate architecture, leading to functional abnormalities and ultimately resulting in leaky membranes, hemorrhage, and high interstitial pressure..$^{12,24}$

VEGF ligands, on binding to their three primary receptors and two co-receptors, initiate signal transduction via dimerization. VEGF receptor 1 is primarily involved in development and is not seen as critical in tumor angiogenesis. VEGF receptor 2 is involved in tumor invasion and migration, while VEGF receptor 3 is innate to lymphatic endothelial cells and plays a role in lymph node metastasis..$^{29,30}$

Clinically, levels of VEGF expression are significantly higher in cervical adenocarcinomas as compared to squamous cell carcinomas. ${ }^{31}$ This may be responsible for the decreased survival and potential for metastatic spread observed for cervical adenocarcinomas as compared to squamous cell carcinomas. ${ }^{32}$ Dobbs et al found that VEGF levels rose with progressive cervical neoplasia from normal tissue 
through intraepithelial neoplasia to invasive carcinomas providing a potential mechanism for stepwise neoplastic transformation. ${ }^{33}$

Heavily pretreated tumors, as is usually the case for metastatic, persistent, or recurrent cervical cancers, have often acquired a variety of mutations that may confer resistance to traditional chemotherapy. Hypoxic tumors have increased VEGF signaling, providing another mechanism for resistance that is targetable by anti-angiogenic drugs. ${ }^{28}$ Paclitaxel resistance is a major hallmark of pretreated advanced cervical cancer, and this has been associated with increased cellular autophagy. By downregulating the HIF-1 alpha pathway, one can decrease VEGF expression as well as autophagy, which can potentially re-sensitize cells to paclitaxel, possibly resulting in improved outcomes. ${ }^{34}$

Novel approaches harnessing the patient's own immune system to improve tumor outcomes are increasingly being utilized across numerous tumor types, including cervical cancer. Recent advances include using HPV specific tumor-infiltrating T-cells has shown regression of metastatic lesions. ${ }^{35}$ Future directions will include synergistic approaches utilizing both targeted therapies such as bevacizumab with immunomodulatory drugs. Alternative combination approaches include using VEGF targeted bevacizumab to target neovascularization, and fosbretabulin as an antivascular agent to attack existing blood vessels, as is being tested in early phase clinical trials. ${ }^{36}$

\section{Data supporting bevacizumab for definitive treatment of cervical cancer}

Randomized Phase III trials combining bevacizumab with chemotherapy and radiation are lacking for cervical cancer in the definitive setting. The initial results of the first cooperative group Phase II trial (RTOG 0417) which evaluated 49 women with bulky Stage IB-IIIB tumors has been reported. ${ }^{37}$ All patients had bevacizumab $(10 \mathrm{mg} / \mathrm{kg}$ every 2 weeks for three cycles) combined with the definitive radiotherapy and concurrent cisplatin, with toxicity as the primary endpoint. Importantly, bevacizumab was delivered for three cycles only, and no maintenance therapy was given.

In the initial publication of RTOG 0417, the gastrointestinal toxicity was relatively mild and only two of the 46 patients developed Grade 3 gastrointestinal adverse events, and no Grade 4 or 5 events were noted. Specifically, there were no gastrointestinal fistulas or perforations noted although concerns initially existed for synergistic effects between radiation and bevacizumab.
All predetermined endpoints were met with hematologic toxicity as the most prevalent among all defined adverse events. ${ }^{37}$ The clinical outcomes, including overall survival, disease-free survival, and loco-regional control, were subsequently reported, showing a 3-year overall survival of $80.2 \% .{ }^{38}$ The 3-year disease-free survival in RTOG 0417 was $68.7 \%$. The 3-year loco-regional failure was $23.2 \%$. In this single arm study, these outcomes compare favorably with historical controls, such as the chemoradiation arm of the landmark RTOG ${ }^{4}$ 90-01. The concept of safely adding bevacizumab to standard chemoradiotherapy was supported in RTOG 0417.

Additional experience combining pelvic radiation with bevacizumab is reported in definitive rectal cancer in which a lower dose ( $5 \mathrm{mg} / \mathrm{kg}$ every 2 weeks) was chosen due to unacceptable dose limiting diarrhea and colitis at dose escalation to $10 \mathrm{mg} / \mathrm{kg}$. While ultimately proving too toxic, the $10 \mathrm{mg} / \mathrm{kg}$ dose of bevacizumab did result in two complete responses in the neoadjuvant treatment of rectal cancer. ${ }^{39}$ Bevacizumab alone has also resulted in substantial bowel toxicity in the treatment of ovarian cancer confirming that this will be the dose limiting toxicity when used solo or in combination with cytotoxic therapies. Potential selection methods by which this toxicity may hopefully be mitigated have been proposed. ${ }^{40}$

One useful aspect of VEGF is the ability to measure circulating levels as a surrogate tumor marker. This has been investigated in both the invasive and preinvasive settings. A change to a hemoangiogenic phenotype from a lymphangiogenic phenotype is associated with transformation to invasion. However, the sensitivity and specificity of these circulating levels are not currently adequate for clinical use at this time. ${ }^{41}$

\section{Data supporting bevacizumab for metastatic, recurrent, or persistent cervical cancer}

Early clinical data, consisting of a small group of six women with metastatic and heavily pretreated cervical cancer, showed a $67 \%$ response rate when combined with cytotoxic chemotherapy. ${ }^{42}$ In this small cohort of patients, bevacizumab was also well tolerated overall, although one patient developed a Grade 4 toxicity (neutropenic sepsis), which was most likely attributable to the cytotoxic component of therapy. Based on early data in patients with few existing options, bevacizumab was introduced in clinical trials.

Monk et al reported the first Phase II trial of bevacizumab in cervical cancer conducted through the Gynecologic 
Oncology Group (GOG). This trial enrolled women with one to two regimens of chemotherapy beyond their initial definitive course with few options for a reasonable chance of response. Bevacizumab was delivered as palliative monotherapy $(15 \mathrm{mg} / \mathrm{kg}$ every 21 days $){ }^{43}$ In this heavily pretreated group, single-agent bevacizumab induced an objective tumor regression in five patients (11\%) and eleven patients $(24 \%)$ were without progressive disease for at least 6 months. Bevacizumab actually outperformed a number of cytotoxic agents when compared with historical single-agent GOG regimens, exceeding trial expectations. ${ }^{44-46}$ Toxicity, as described earlier, included hypertension, venous thromboembolic disease, and gastroenteritis, and generally did not lead to stopping therapy early.

GOG 240 was initiated based on these impressive results. This trial evaluated chemotherapy with and without codelivery of concurrent bevacizumab. ${ }^{14}$ In this four-arm $2 \times 2$ factorial design trial, bevacizumab was given at the same dose as in the Phase II trial $(15 \mathrm{mg} / \mathrm{kg})$ with and without two separate chemotherapy doublets, either cisplatin plus paclitaxel or topotecan plus paclitaxel. Initial planned analysis demonstrated that the topotecan-containing doublet was not superior to the standard doublet with cisplatin. Overall survival was improved by 3.7 months in GOG 240 (17 versus 13.3 months) for those who received bevacizumab regardless of their concomitant doublet backbone. Overall response rates were also higher in those patients receiving bevacizumab as a component of therapy (48\% versus $36 \%)$. Toxicity was consistent with previous bevacizumab experience, including hypertension (25\%), venous thromboembolic events (8\%), and gastrointestinal fistulas (3\%). Carboplatin and paclitaxel are considered the standard of care for gynecologic malignancies based on their favorable toxicity profile. ${ }^{47}$ This trial demonstrated similar outcomes to the metastatic first ${ }^{48}$ and second line ${ }^{49}$ colorectal as well as non-small-cell lung cancer trials, ${ }^{50}$ confirming a significant benefit to bevacizumab in heavily pretreated patients. Quality of life has been carefully analyzed and reported from GOG 240, showing no overall decrement in quality of life. ${ }^{51}$ This analysis used a summary index, brief pain inventory, and neurotoxicity analysis to delve into the quality of life on bevacizumab. While some suggestion of a slight difference in neurotoxicity is noted when group means are compared, the difference is not significant, providing a more robust comparison and further reassurance that there are no significant side effects from adding bevacizumab to chemotherapy. ${ }^{52}$
Recent trials have focused on pairing bevacizumab with other agents that can rationally increase the response rate and clinical outcomes, while maintaining acceptable toxicity. A Phase I trial investigated the combination of VEGF targeted bevacizumab with an agent from an antivascular class that can better target existing vasculature (versus neovascularization). ${ }^{36}$ This demonstrated a 39\% reduction in vascularization which persisted over time and has led to an open Phase II trial testing bevacizumab monotherapy versus the bevacizumabfosbretabulin combination in recurrent or metastatic ovarian cancer with potential future applications in additional gynecologic malignancies, including cervical cancer.

\section{Conclusion}

Clinical outcomes have been disappointing in metastatic, persistent, and recurrent cervical cancer, despite utilization of the best chemotherapy and radiation regimens applied. Current and future studies are rationally applying targeted therapy in addition to the best local and systemic therapy. Bevacizumab represents an early example of this progress in cervical cancer, demonstrating an overall survival benefit with chemotherapy without a decrement in quality of life compared to chemotherapy alone. ${ }^{14}$ Additionally, given the survival benefit observed in GOG 240 among patients previously treated with chemoradiation, one could envision an adjuvant role for bevacizumab in maintaining excellent outcomes in the high-risk cervical cancer population treated definitively. A Phase III trial would be appropriate to address this possibility and clarify the risks and benefits in this patient subset.

Improved outcomes for metastatic patients are vitally important, yet improved control in curative patients, is equally promising. While caution must be applied in overlapping therapies with chemotherapy and radiation used to in the definite setting, it is clear that targeted therapy represents a promising approach for improved outcomes. Further trials are underway attempting to do just this. ${ }^{53}$

National guidelines targeting oncologists have upgraded the recommendations for bevacizumab to a first line option. GOG 240 met its primary endpoints without a significant decrement in quality of life, leading to a new standard for advanced cervical cancer incorporating bevacizumab with chemotherapy. ${ }^{54}$ The US FDA approved bevacizumab for recurrent cervical cancer in 2014 as the first anti-angiogenic therapy in gynecologic malignancies to demonstrate an overall survival benefit. ${ }^{55}$ The most recent version of the National Comprehensive Cancer Network guidelines, published in 
2015, endorses cisplatin-paclitaxel-bevacizumab as well as topotecan-paclitaxel-bevacizumab as first line combination therapy for recurrent or metastatic cervical cancer. ${ }^{56}$ These changes will help drive clinical practice across the country.

Practice patterns studies, available in this era of big data, reflect changing trends in local therapy based on trial results. ${ }^{57}$ Future practice patterns data will likely reflect changing practice to incorporate bevacizumab as standard of care for recurrent and metastatic patients. Additionally, the development of nomograms to help predict outcomes such as overall survival, pelvic recurrence, and progression-free survival can help clinicians have better conversations about the potential benefits of bevacizumab in different clinical scenarios. ${ }^{58}$ Given our rapidly increasing knowledge of cervical cancer biology, predictive biomarkers, ${ }^{59,60}$ patient nomograms, ${ }^{58}$ and functional imaging, ${ }^{61}$ these strategies will play an important role in future patient selection for therapies utilizing immune, ${ }^{35}$ targeted, ${ }^{14}$ and combination targeted therapy approaches. ${ }^{13}$

One additional concern with the increasing utilization of bevacizumab is related to the cost of care. Despite an overall survival benefit, this therapy will have limited availability in many of the countries with the highest burden of cervical cancer. ${ }^{62}$ On Markov model analysis, the costs are primarily related to the drug, which may make this a cost-effective treatment only when biosimilars or lower cost agents are available. $^{63}$

But bevacizumab in cervical cancer is a prime example of how a rational application of targeted therapy costs in terms of additional toxicity in susceptible tumors can lead to marked improvement in outcomes. Cost modeling supports the effectiveness of this intervention, particularly when lower cost biosimilars are available. Finally, the reports of RTOG 0417 now offer prospective multi-institutional evidence of the feasibility and safety when bevacizumab is administered in addition to standard chemoradiation. However, the role of bevacizumab in the definitive setting with chemoradiation remains to be determined.

\section{Disclosure}

The authors report no conflicts of interest in this work.

\section{References}

1. Siegel RL, Miller KD, Jemal A. Cancer statistics, 2015. CA Cancer J Clin. 2015;65(1):5-29.

2. Global Burden of Disease Cancer Collaboration, Fitzmaurice C, Dicker D, et al. The Global Burden of Cancer 2013. JAMA Oncol. 2015;1(4): 505-527.
3. Duenas-Gonzalez A, Orlando M, Zhou Y, Quinlivan M, Barraclough H. Efficacy in high burden locally advanced cervical cancer with concurrent gemcitabine and cisplatin chemoradiotherapy plus adjuvant gemcitabine and cisplatin: prognostic and predictive factors and the impact of disease stage on outcomes from a prospective randomized phase III trial. Gynecol Oncol. 2012;126(3):334-340.

4. Morris M, Eifel PJ, Lu J, et al. Pelvic radiation with concurrent chemotherapy compared with pelvic and para-aortic radiation for high-risk cervical cancer. $N$ Engl J Med. 1999;340(15):1137-1143.

5. Ignoffo RJ. Overview of bevacizumab: a new cancer therapeutic strategy targeting vascular endothelial growth factor. Am J Health Syst Pharm. 2004;61(21 Suppl 5):S21-S26.

6. Braghiroli MI, Sabbaga J, Hoff PM. Bevacizumab: overview of the literature. Expert Rev Anticancer Ther. 2012;12(5):567-580.

7. Jain RK. Normalization of tumor vasculature: an emerging concept in antiangiogenic therapy. Science. 2005;307(5706):58-62.

8. Jain RK, Duda DG, Clark JW, Loeffler JS. Lessons from phase III clinical trials on anti-VEGF therapy for cancer. Nat Clin Pract Oncol. 2006; 3(1):24-40.

9. Lee CG, Heijn M, di Tomaso E, et al. Anti-Vascular endothelial growth factor treatment augments tumor radiation response under normoxic or hypoxic conditions. Cancer Res. 2000;60(19):5565-5570.

10. Teicher BA, Dupuis N, Kusomoto T, et al. Antiangiogenic agents can increase tumor oxygenation and response to radiation therapy. Radiat Oncol Investig. 1994;2(6):269-276.

11. Bennouna J, Sastre J, Arnold D, et al. Continuation of bevacizumab after first progression in metastatic colorectal cancer (ML18147): a randomised phase 3 trial. Lancet Oncol. 2013;14(1):29-37.

12. Goel S, Duda DG, Xu L, et al. Normalization of the vasculature for treatment of cancer and other diseases. Physiol Rev. 2011;91(3):1071-1121.

13. Jackson MW, Rusthoven CG, Fisher CM, Schefter TE. Clinical potential of bevacizumab in the treatment of metastatic and locally advanced cervical cancer: current evidence. OncoTargets Ther. 2014;7:751-759.

14. Tewari KS, Sill MW, Long HJ, et al. Improved Survival with Bevacizumab in advanced cervical cancer. $N$ Engl J Med. 2014;370(8):734-743.

15. Walboomers JM, Jacobs MV, Manos MM, et al. Human papillomavirus is a necessary cause of invasive cervical cancer worldwide. J Pathol. 1999;189(1):12-19.

16. Rodriguez-Carunchio L, Soveral I, Steenbergen RD, et al. HPV-negative carcinoma of the uterine cervix: a distinct type of cervical cancer with poor prognosis. BJOG. 2015;122(1):119-127.

17. Yang X, Lu L. Expression of HPV-16 E6 protein and p53 inactivation increases the uterine cervical cancer invasion. Drug Res (Stuttg). 2015;65(2):70-73.

18. Birner P, Schindl M, Obermair A, Plank C, Breitenecker G, Oberhuber G. Overexpression of hypoxia-inducible factor $1 \alpha$ is a marker for an unfavorable prognosis in early-stage invasive cervical cancer. Cancer Res. 2000;60(17):4693-4696.

19. Mukhopadhyay D, Tsiokas L, Sukhatme VP. Wild-type p53 and v-Src exert opposing influences on human vascular endothelial growth factor gene expression. Cancer Res. 1995;55(24):6161-6165.

20. Ghahremani MF, Goossens S, Nittner D, et al. p53 promotes VEGF expression and angiogenesis in the absence of an intact $\mathrm{p} 21-\mathrm{Rb}$ pathway. Cell Death Differ. 2013;20(7):888-897.

21. Huang M, Chen Q, Xiao J, et al. Overexpression of hypoxia-inducible factor-1alpha is a predictor of poor prognosis in cervical cancer: a clinicopathologic study and a meta-analysis. Int J Gynecol Cancer. 2014; 24(6):1054-1064.

22. Fu SL, Miao J, Ding B, et al. A polymorphism in the $3^{\prime}$ untranslated region of Hypoxia-Inducible Factor-1 alpha confers an increased risk of cervical cancer in a Chinese population. Neoplasma. 2014;61(1):63-69.

23. Ferrara N, Hillan KJ, Gerber HP, Novotny W. Discovery and development of bevacizumab, an anti-VEGF antibody for treating cancer. Nat Rev Drug Discov. 2004;3(5):391-400.

24. Carmeliet P. VEGF as a key mediator of angiogenesis in cancer. Oncology. 2005;69(Suppl 3):4-10. 
25. Raspollini M, Amunni G, Villanucci A, Baroni G, Boddi V, Taddei G. Prognostic significance of microvessel density and vascular endothelial growth factor expression in advanced ovarian serous carcinoma. Int J Gynecol Cancer. 2004;14(5):815-823.

26. Dellas A, Moch H, Schultheiss E, et al. Angiogenesis in cervical neoplasia: microvessel quantitation in precancerous lesions and invasive carcinomas with clinicopathological correlations. Gynecol Oncol. 1997; 67(1):27-33.

27. Cooper RA, Wilks DP, Logue JP, et al. High tumor angiogenesis is associated with poorer survival in carcinoma of the cervix treated with radiotherapy. Clin Cancer Res. 1998;4(11):2795-2800.

28. Byrne AM, Bouchier-Hayes D, Harmey J. Angiogenic and cell survival functions of vascular endothelial growth factor (VEGF). J Cell Mol Med. 2005;9(4):777-794.

29. Hicklin DJ, Ellis LM. Role of the vascular endothelial growth factor pathway in tumor growth and angiogenesis. J Clin Oncol. 2005;23(5):1011-1027.

30. Rini BI, Small EJ. Biology and clinical development of vascular endothelial growth factor-targeted therapy in renal cell carcinoma. J Clin Oncol. 2005;23(5):1028-1043.

31. Tokumo K, Kodama J, Seki N, et al. Different angiogenic pathways in human cervical cancers. Gynecol Oncol. 1998;68(1):38-44.

32. Eifel PJ, Burke TW, Morris M, Smith TL. Adenocarcinoma as an independent risk factor for disease recurrence in patients with stage IB cervical carcinoma. Gynecol Oncol. 1995;59(1):38-44.

33. Dobbs S, Hewett P, Johnson I, Carmichael J, Murray J. Angiogenesis is associated with vascular endothelial growth factor expression in cervical intraepithelial neoplasia. Br J Cancer. 1997;76(11):1410-1415.

34. Peng X, Gong F, Chen Y, et al. Autophagy promotes paclitaxel resistance of cervical cancer cells: involvement of Warburg effect activated hypoxia-induced factor 1-alpha-mediated signaling. Cell Death Dis. 2014;5:e1367.

35. Stevanovic S, Draper LM, Langhan MM, et al. Complete regression of metastatic cervical cancer after treatment with human papillomavirus-targeted tumor-infiltrating T cells. J Clin Oncol. 2015;33(14):1543-1550.

36. Nathan $P$, Zweifel M, Padhani AR, et al. Phase I trial of combretastatin A4 phosphate (CA4P) in combination with bevacizumab in patients with advanced cancer. Clin Cancer Res. 2012;18(12):3428-3439.

37. Schefter TE, Winter K, Kwon JS, et al. A phase II study of bevacizumab in combination with definitive radiotherapy and cisplatin chemotherapy in untreated patients with locally advanced cervical carcinoma: preliminary results of RTOG 0417. Int J Radiat Oncol Biol Phys. 2012; 83(4):1179-1184.

38. Schefter T, Winter K, Kwon JS, et al. RTOG 0417: Efficacy of bevacizumab in combination with definitive radiation therapy and cisplatin chemotherapy in untreated patients with locally advanced cervical carcinoma. Int J Radiat Oncol Biol Phys. 2014;88(1):101-105.

39. Willett CG, Boucher Y, Duda DG, et al. Surrogate markers for antiangiogenic therapy and dose-limiting toxicities for bevacizumab with radiation and chemotherapy: continued experience of a phase I trial in rectal cancer patients. J Clin Oncol. 2005;23(31):8136-8139.

40. Simpkins F, Belinson JL, Rose PG. Avoiding bevacizumab related gastrointestinal toxicity for recurrent ovarian cancer by careful patient screening. Gynecol Oncol. 2007;107(1):118-123.

41. Kuemmel S, Thomas A, Landt S, et al. Circulating vascular endothelial growth factors and their soluble receptors in pre-invasive, invasive and recurrent cervical cancer. Anticancer Res. 2009;29(2):641-645.

42. Wright JD, Viviano D, Powell MA, et al. Bevacizumab combination therapy in heavily pretreated, recurrent cervical cancer. Gynecol Oncol. 2006;103(2):489-493.

43. Monk BJ, Sill MW, Burger RA, Gray HJ, Buekers TE, Roman LD. Phase II trial of bevacizumab in the treatment of persistent or recurrent squamous cell carcinoma of the cervix: a gynecologic oncology group study. J Clin Oncol. 2009;27(7):1069-1074.

44. Rose PG, Blessing JA, Mayer AR, Homesley HD. Prolonged oral etoposide as second-line therapy for platinum-resistant and platinumsensitive ovarian carcinoma: a Gynecologic Oncology Group study. J Clin Oncol. 1998;16(2):405-410.
45. Bookman MA, Blessing JA, Hanjani P, Herzog TJ, Andersen WA. Topotecan in squamous cell carcinoma of the cervix: a phase II study of the Gynecologic Oncology Group. Gynecol Oncol. 2000;77(3):446-449.

46. Mannel RS, Blessing JA, Boike G. Cisplatin and pentoxifylline in advanced or recurrent squamous cell carcinoma of the cervix: a phase II trial of the Gynecologic Oncology Group. Gynecol Oncol. 2000;79(1):64-66.

47. Kitagawa R, Katsumata N, Shibata T, et al. A randomized, phase III trial of paclitaxel plus carboplatin (TC) versus paclitaxel plus cisplatin (TP) in stage IVb, persistent or recurrent cervical cancer: Japan Clinical Oncology Group study (JCOG 0505). J Clin Oncol. 2012;30(15 suppl):5006.

48. Hurwitz H, Fehrenbacher L, Novotny W, et al. Bevacizumab plus irinotecan, fluorouracil, and leucovorin for metastatic colorectal cancer. N Engl J Med. 2004;350(23):2335-2342.

49. Giantonio BJ, Catalano PJ, Meropol NJ, et al. Bevacizumab in combination with oxaliplatin, fluorouracil, and leucovorin (FOLFOX4) for previously treated metastatic colorectal cancer: results from the Eastern Cooperative Oncology Group Study E3200. J Clin Oncol. 2007; 25(12):1539-1544.

50. Sandler A, Gray R, Perry MC, et al. Paclitaxel-carboplatin alone or with bevacizumab for non-small-cell lung cancer. $N$ Engl $J$ Med. 2006;355(24):2542-2550.

51. Penson RT, Huang HQ, Wenzel LB, et al. Bevacizumab for advanced cervical cancer: patient-reported outcomes of a randomised, phase 3 trial (NRG Oncology-Gynecologic Oncology Group protocol 240). Lancet Oncol. 2015;16(3):301-311.

52. Cella D. Bevacizumab and quality of life in advanced cervical cancer. Lancet Oncol. 2015;16(3):241-243.

53. Lheureux S, Butler MO, Fleming GF, et al. A phase I/II study of ipilimumab in women with metastatic or recurrent HPV-related cervical carcinoma. J Clin Oncol. 2014;32(5s):5631.

54. Eskander RN, Tewari KS. Development of bevacizumab in advanced cervical cancer: pharmacodynamic modeling, survival impact and toxicology. Future Oncol. 2015;11(6):909-922.

55. Liu FW, Cripe J, Tewari KS. Anti-angiogenesis therapy in gynecologic malignancies. Oncology (Williston Park). 2015;29(5):350-360.

56. Koh WJ, Greer BE, Abu-Rustum NR, et al. Cervical Cancer, Version 2.2015. J Nati Compr Canc Netw. 2015;13(4):395-404; quiz 404.

57. Carlson JA, Rusthoven C, DeWitt PE, Davidson SA, Schefter TE, Fisher CM. Are we appropriately selecting therapy for patients with cervical cancer? Longitudinal patterns-of-care analysis for stage IB-IIB cervical cancer. Int J Radiat Oncol Biol Phys. 2014;90(4):786-793.

58. Rose PG, Java J, Whitney CW, et al. Nomograms Predicting Progression-Free Survival, Overall Survival, and Pelvic Recurrence in Locally Advanced Cervical Cancer Developed From an Analysis of Identifiable Prognostic Factors in Patients From NRG Oncology/Gynecologic Oncology Group Randomized Trials of Chemoradiotherapy. J Clin Oncol. 2015;39(19):2136-2142.

59. Jubb AM, Harris AL. Biomarkers to predict the clinical efficacy of bevacizumab in cancer. Lancet Oncol. 2010;11(12):1172-1183.

60. Lambrechts D, Lenz HJ, de Haas S, Carmeliet P, Scherer SJ. Markers of response for the antiangiogenic agent bevacizumab. J Clin Oncol. 2013; 31(9):1219-1230.

61. Kidd EA, Siegel BA, Dehdashti F, Grigsby PW. Pelvic lymph node F-18 fluorodeoxyglucose uptake as a prognostic biomarker in newly diagnosed patients with locally advanced cervical cancer. Cancer. 2010;116(6):1469-1475.

62. Denny LA. Questions we should be asking about bevacizumab for cervical cancer. Oncology (Williston Park). 2015;29(5):367-368.

63. Minion LE, Bai J, Monk BJ, et al. A Markov model to evaluate cost-effectiveness of antiangiogenesis therapy using bevacizumab in advanced cervical cancer. Gynecol Oncol. 2015;137(3):490-496. 
OncoTargets and Therapy

\section{Publish your work in this journal}

OncoTargets and Therapy is an international, peer-reviewed, open access journal focusing on the pathological basis of all cancers, potential targets for therapy and treatment protocols employed to improve the management of cancer patients. The journal also focuses on the impact of management programs and new therapeutic agents and protocols on

patient perspectives such as quality of life, adherence and satisfaction. The manuscript management system is completely online and includes a very quick and fair peer-review system, which is all easy to use. Visit http://www.dovepress.com/testimonials.php to read real quotes from published authors.

Submit your manuscript here: http://www.dovepress.com/oncotargets-and-therapy-journal 